\title{
Dual Circularly Polarized Omnidirectional Antenna with Slot Array on Coaxial Cylinder
}

\author{
Bin Zhou, ${ }^{1}$ Junping Geng, ${ }^{1}$ Zhe Li, ${ }^{2}$ Wenzhi Wang, ${ }^{1}$ Xianling Liang, ${ }^{1}$ and Ronghong Jin' \\ ${ }^{1}$ Department of Electronic Engineering, Shanghai Jiao Tong University, Shanghai 200240, China \\ ${ }^{2}$ China Academy of Launch Vehicle Technology, Beijing 100076, China \\ Correspondence should be addressed to Junping Geng; gengjunp@sjtu.edu.cn
}

Received 26 May 2015; Revised 13 July 2015; Accepted 15 July 2015

Academic Editor: Xiulong Bao

Copyright (c) 2015 Bin Zhou et al. This is an open access article distributed under the Creative Commons Attribution License, which permits unrestricted use, distribution, and reproduction in any medium, provided the original work is properly cited.

A dual circularly polarized (CP) omnidirectional antenna based on slot array in coaxial cylinder structure is presented in this paper. It is constructed by perpendicular slot pairs around and along the axis of the coaxial cylinder to realize the omnidirectional CP property, and two ports are assigned in its two sides as left hand circularly polarized (LHCP) port and right hand circularly polarized (RHCP) port, respectively. The proposed antenna achieves a bandwidth of $16.4 \%$ ranging from 5.05 to $5.95 \mathrm{GHz}$ with an isolation higher than $15 \mathrm{~dB}$ between the two CP ports, and the return loss (RL) is higher than $10 \mathrm{~dB}$ within the bandwidth in both of the two ports. From the measured results, the average axial ratio (AR) of the proposed antenna in omnidirectional plane is lower than $1.5 \mathrm{~dB}$.

\section{Introduction}

For a RF receiver which needs to receive electromagnetic signals with any polarizations mode and from any directions on the ground, the dual CP omnidirectional antenna is significant and valuable. The dual CP omnidirectional antenna is widely used, such as wireless communications, radio broadcasting, and navigation radar [1-3]. In recent years, there are many researches about omnidirectional antenna and CP antenna. In [4], a miniaturization of omnidirectional $\mathrm{CP}$ antenna relies on folding the antennas patch underneath itself, decreasing overall footprint, which is small, but with a narrow bandwidth of only $0.6 \%(2.392-2.407 \mathrm{GHz})$ and the axial ratio in some directions of the omnidirectional plane is higher than $3 \mathrm{~dB}$. For the antenna proposed in [5], by introducing several inclined slits to the diagonal and sidewalls of the rectangular dielectric resonator (RDR) and also deducting a rectangular part of the top wall of the linearly polarized (LP) rectangular dielectric resonator antenna (RDRA), degeneracy modes are excited to generate the circularly polarized (CP) fields, while the bandwidth is narrow and the gain fluctuation in the omnidirectional plane is higher than $5 \mathrm{~dB}$ from the results of the radiation patterns, so it does not perform well in the omnidirectional character.
Literature [6] reports a dual CP antenna which is excited at the second-order mode to generate the conical radiation pattern and is fed by a hybrid coupler to obtain the dual $\mathrm{CP}$ operation, but it realizes a conical beam instead of an omnidirectional coverage radiation pattern. There are only a few researches about omnidirectional dual CP antenna. In literature [7], an omnidirectional dual-band dual circularly polarized microstrip antenna is proposed, while the dual circularly polarization means that the antenna provides different single RHCP and LHCP in its two different bandwidths, respectively, so it is single circularly polarized antenna in the specific frequency bandwidth actually. In [8], a dual-CP omnidirectional antenna is proposed, which consists of 4 tilted dipoles with parasitic elements for each sense of circular polarization.

In this paper, an omnidirectional dual CP coaxial slot array antenna with perpendicular slot pairs around and along the axis of the out conductor is presented. The RHCP port and LHCP port are designed in the two sides of the antenna, respectively. The proposed antenna provides a wide bandwidth and, within the bandwidth, it performs well with good axial ratio property and omnidirectional property. Section 2 presents the antenna structure and design principle. Section 3 discusses the measured results, including 


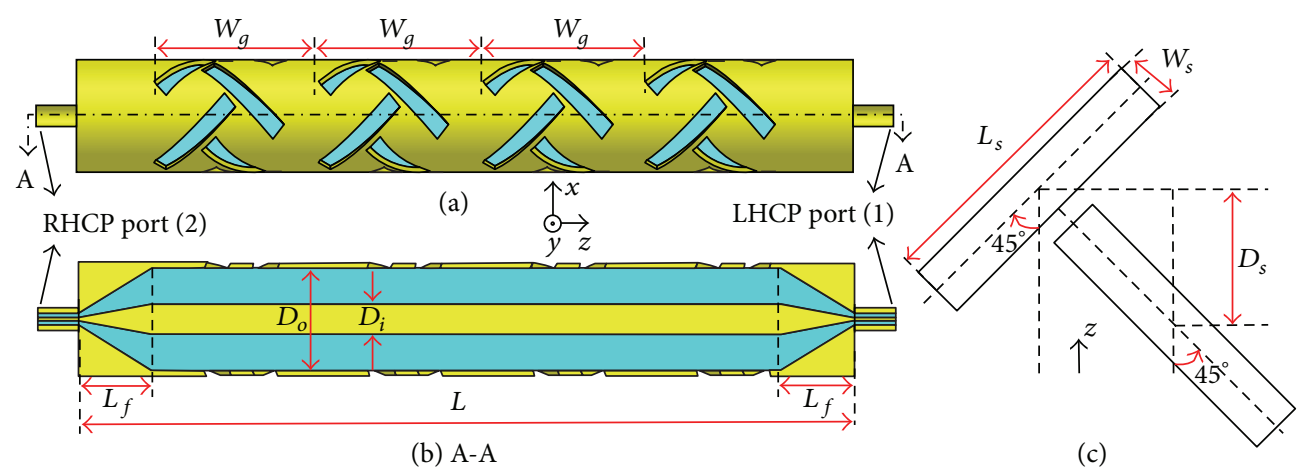

Figure 1: Geometry of the omnidirectional dual circularly polarized (CP) antenna. (a) Side view. (b) A-A section view. (c) Geometry of the slot pairs.

bandwidth, radiation pattern, and axial ratio (AR). Section 4 gives the conclusions.

\section{Antenna Design}

2.1. Antenna Structure. As shown in Figure 1, the proposed dual CP antenna omnidirectional in this paper is based on the single $\mathrm{CP}$ omnidirectional antenna represented in literature [9] constructed with coaxial cylinder structure. Two ports are assigned in the two sides of the coaxial cylinder as left hand circularly polarized (LHCP) port and right hand circularly polarized (RHCP) port, respectively. The characteristic impedance of the coaxial cylinder is designed as $50 \Omega$ in this paper, which is the same as the characteristic impedance of the two SMA adaptor ports. The coaxial tapered line is in both of the two sides between the slot arrays and the two SMA adaptor ports, and it achieves the goal of port match.

The basic radiation elements are slots in the out conductor of the coaxial cylinder. There are four circles of slots along the axis of the coaxial cylinder. Each circle of slots includes 8 slots which are perpendicular to each other and symmetrically arranged around the coaxial cylinder axis in the out conductor. Every circle of the slots can be seen as four perpendicular slot pairs around the axis.

The structure of the perpendicular slot pairs is shown in Figure 2. Each of the slots leans an angle of 45 degrees with the axis of the coaxial cylinder and the interval of the two perpendicular slots is $\lambda_{g} / 4$ ( $\lambda_{g}$ is the medium wavelength) along the feed direction. In that case, the electric fields radiated from the slot pairs are vertical with each other and have a 90-degree phase difference, so the circularly polarized wave is generated. For the distance of the $\lambda_{g} / 4$ between the adjacent pairs of slots, the reflection power from radiation slots back to the feeding point is cancelled. As slot antenna, the slot in the out conductor of the coaxial cylinder can be equivalent as magnetic dipole with a length of $\lambda_{g} / 2$ in the vertical direction with the axis. Four of the slot pairs symmetrically surrounding the coaxial cylinder axis achieve the omnidirectional coverage character. Four circles of the slot pairs, which are identical with each other, are arranged along the axis to constitute the slot array which will provide a higher gain. To achieve the omnidirectional pattern,
TABLE 1: Optimized geometric parameters of the omnidirectional $\mathrm{CP}$ antenna.

\begin{tabular}{lccc}
\hline$L$ & $153.5 \mathrm{~mm}$ & $W_{g}$ & $40 \mathrm{~mm}$ \\
$D_{i}$ & $7.6 \mathrm{~mm}$ & $D_{o}$ & $28 \mathrm{~mm}$ \\
$L_{s}$ & $24.4 \mathrm{~mm}$ & $W_{s}$ & $4.3 \mathrm{~mm}$ \\
$D_{s}$ & $11.8 \mathrm{~mm}$ & $L_{f}$ & $18 \mathrm{~mm}$ \\
\hline
\end{tabular}

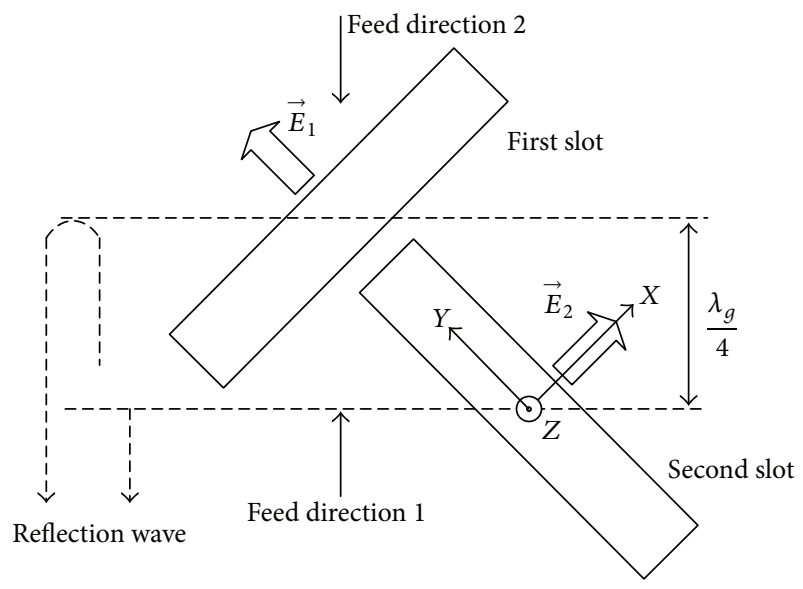

FIGURE 2: The adjacent perpendicular slot pairs.

the electromagnetic wave radiated from every circle of slots should be the same phases, so the four circles of the slot are arranged with a distance of $\lambda_{g}$.

It is a symmetrical structure antenna. The thickness of the out conductor of the coaxial cylinder is $t=1.3 \mathrm{~mm}$, and the space between the out conductor and the inner conductor of the coaxial cylinder is filled with Teflon medium with a permittivity of $\varepsilon_{r}=2.1$. The proposed antenna is simulated and optimized by CST and the optimized geometric parameters are summarized in Table 1.

2.2. Dual CP Principle. Figure 2 shows two adjacent slots from all the slots in the out conductor, which are perpendicular to each other. The reference coordinate system is created with the slot pairs, whose $y$ - and $x$-axes are the same direction with the polarization of the electric fields radiated by the two slots, respectively, and the $+z$-axis is the same direction 
with the propagation direction of the electric fields $[10,11]$. It is assumed that the total electric fields are $\vec{E}$ and the fields radiated from the first slot and the second slot are $\vec{E}_{1}$ and $\vec{E}_{2}$, respectively. Then their relation is

$$
\vec{E}=\vec{E}_{1}+\vec{E}_{2}=E_{x} \vec{a}_{x}+E_{y} \vec{a}_{y} .
$$

For the distance of the two slots, it is supposed that the amplitude of the $E_{x}$ and $E_{y}$ is $E_{x 0}$ and $E_{y 0}\left(E_{x 0}>0, E_{y 0}>0\right)$, and, compared to $E_{x}, E_{y}$ is laggard in phase with a difference of $\varphi$. Then (1) can be changed as

$$
\begin{aligned}
\vec{E}(z, t)= & E_{x 0} \cos (\omega t-k z) \vec{a}_{x} \\
& +E_{y 0} \cos (\omega t-k z-\varphi) \vec{a}_{y},
\end{aligned}
$$

where

$$
\begin{aligned}
& E_{x}(z, t)=E_{x 0} \cos (\omega t-k z), \\
& E_{y}(z, t)=E_{y 0} \cos (\omega t-k z-\varphi) .
\end{aligned}
$$

The relation between $E_{x}(z, t)$ and $E_{y}(z, t)$ can be concluded from (3):

$$
\begin{aligned}
& {\left[\frac{E_{x}(z, t)}{E_{x 0}}\right]^{2}+\left[\frac{E_{y}(z, t)}{E_{y 0}}\right]^{2}-\frac{2 E_{x}(z, t) E_{y}(z, t)}{E_{x 0} E_{y 0}} \cos \varphi} \\
& \quad=\sin ^{2} \varphi .
\end{aligned}
$$

Since the distance of the two slots in the feed direction is $\lambda_{g} / 4$, the phase difference is $\varphi= \pm 90^{\circ}$. It is supposed that the amplitude of electric fields is $E_{x 0}=E_{y 0}=E_{0}$; then from (4)

$$
E_{x}^{2}(z, t)+E_{y}^{2}(z, t)=E_{0}^{2} .
$$

It can be seen from (3) that the amplitude of $\vec{E}(z, t)$ will not change with time $t$, and the angles between the polarization direction of $\vec{E}(z, t)$ and $+x$-axis direction are

$$
\begin{aligned}
\alpha & =\arctan \frac{E_{0} \cos (\omega t-k z \pm \pi / 2)}{E_{0} \cos (\omega t-k z)} \\
& =\arctan [\mp \tan (\omega t-k z)]=\mp(\omega t-k z) .
\end{aligned}
$$

In any positions, $z$ is a constant, and the polarized vector direction of the field $\vec{E}(z, t)$ is rotary with a constant angular frequency $\omega$ by the increasing of $t$. As shown in Figure 2, when excited as fed direction 1, the phase of $E_{y}$ is laggard compared to the phase of $E_{x}$, so $\varphi=90^{\circ}$ and $\alpha=\omega t$. The electric fields vector direction is anticlockwise, so it is right hand circularly polarized (RHCP) wave. When excited as fed direction 2, the phase of $E_{y}$ exceeds the phase of $E_{x}$, so $\varphi=-90^{\circ}$ and $\alpha=-\omega t$. The electric fields vector direction is clockwise, so it is left hand circularly polarized (LHCP) wave. Comparing Figure 2 with the slots distribution in Figure 1, the RHCP port and LHCP port are corresponding with fed direction 1 and fed direction 2 , respectively.

For the different distances of the two slots relative to the fed port, the electric fields amplitude of the two slots is unequal $\left(E_{x 0} \neq E_{y 0}\right)$. This is an influence factor of the axial ratio and their relation is reflected by the axial ratio character of the antenna.

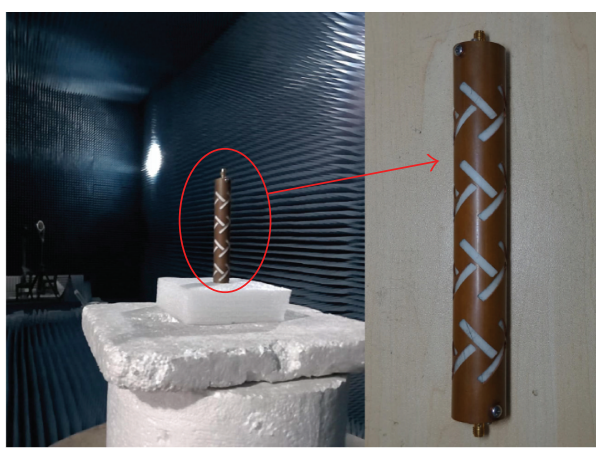

FIGURE 3: Photograph of the omnidirectional CP antenna being tested in the anechoic chamber.

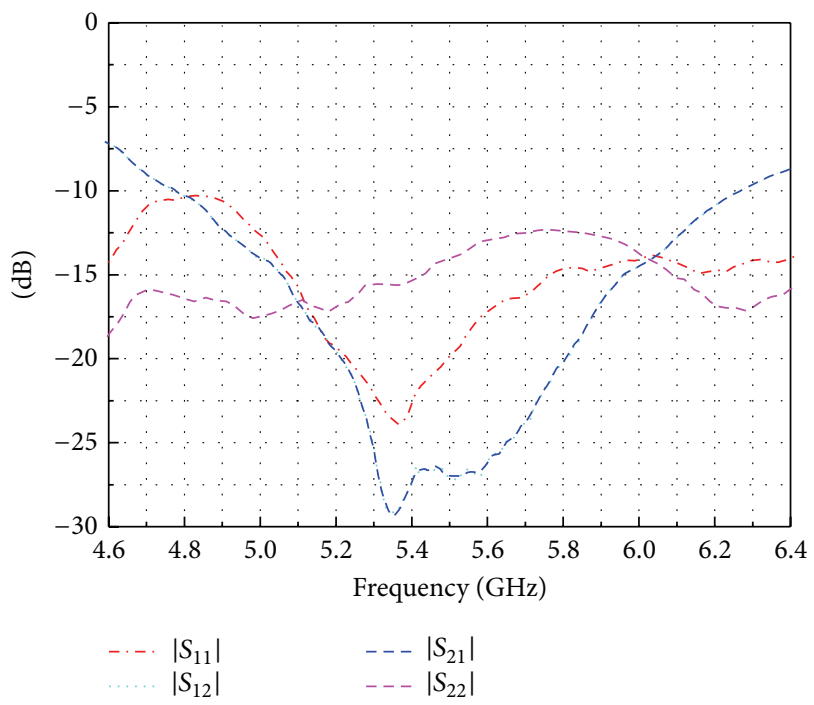

Figure 4: Measured S-parameters results.

\section{Results and Discussion}

Simulated and measured results of the proposed antenna are presented in this section. To measure the LHCP property and RHCP property of the antenna, there are two steps to do. When port 1 (LHCP port) is excited and port 2 (RHCP port) is terminated with a $50 \Omega$ load, the antenna generates the LHCP radiation and we get LHCP results, whereas when port 2 is excited and port 1 is terminated with a $50 \Omega$ load, the antenna generates the RHCP radiation and we get RHCP measurement results. Figure 3 shows the antenna structure and the test scenario of the omnidirectional dual CP antenna.

The measured $S$-parameters results are shown in Figure 4. The proposed antenna achieves a bandwidth of $16.4 \%$ at $5.05 \sim$ $5.95 \mathrm{GHz}$, during which bandwidth the isolation between the two ports is higher than $15 \mathrm{~dB}$, and both of the measured $\left|S_{11}\right|$ and $\left|S_{22}\right|$ values are lower than $-10 \mathrm{~dB}$. The reflection of the two ports is small and the isolation between the two ports is high, which reflects that the antenna works well with two polarizations.

Figure 5 shows the simulated and measured normalized copolarization and cross-polarization radiation patterns 

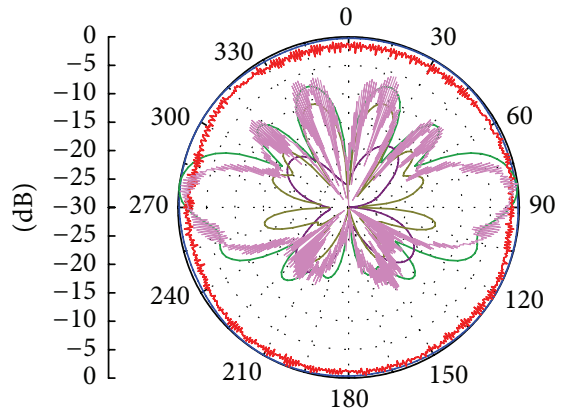

_ $x y$-plane simulated copolarization

_ $x z$-plane simulated copolarization

- $x y$-plane simulated cross-polarization

_ $x z$-plane simulated cross-polarization

$x y$-plane measured copolarization

$x z$-plane measured copolarization

(a)

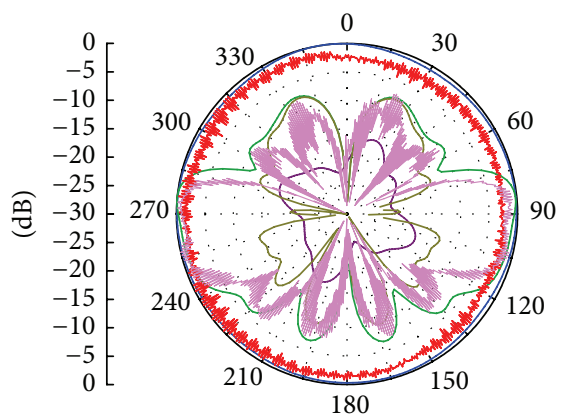

$x y$-plane simulated copolarization
$x z$-plane simulated copolarization
$x y$-plane simulated cross-polarization
$x z$-plane simulated cross-polarization
$\quad x y$-plane measured copolarization
$x z$-plane measured copolarization

(b)
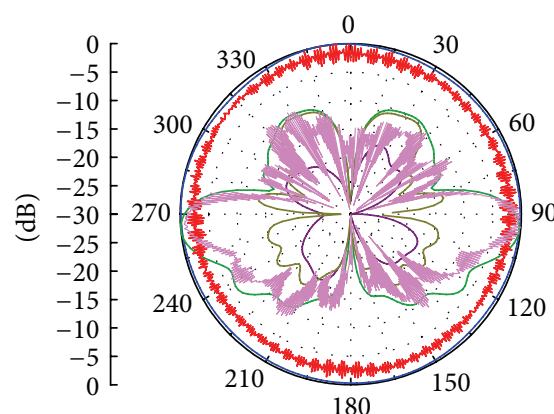

$-x y$-plane simulated copolarization
$x z$-plane simulated copolarization
$\quad x y$-plane simulated cross-polarization
$\quad x z$-plane simulated cross-polarization
$x y$-plane measured copolarization
$x z$-plane measured copolarization

(c)
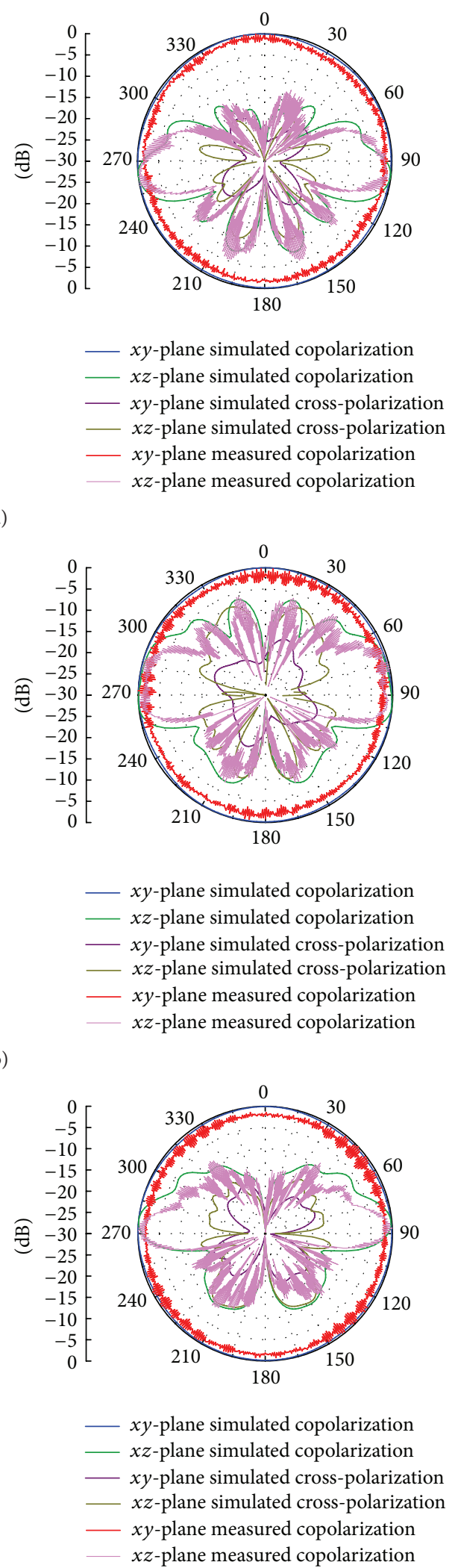

FIGURE 5: Simulated and measured LHCP (in left) and RHCP (in right) normalized copolarization and cross-polarization radiation patterns in the $x y$-plane (omnidirectional plane) and $x z$-plane. (a) $5.1 \mathrm{GHz}$, (b) $5.5 \mathrm{GHz}$, and (c) $5.9 \mathrm{GHz}$. 


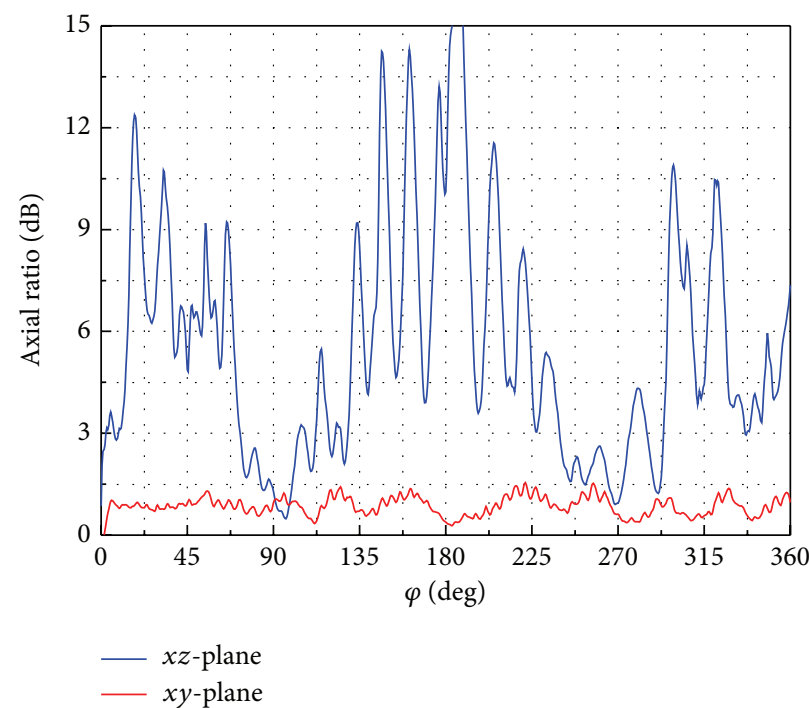

(a)

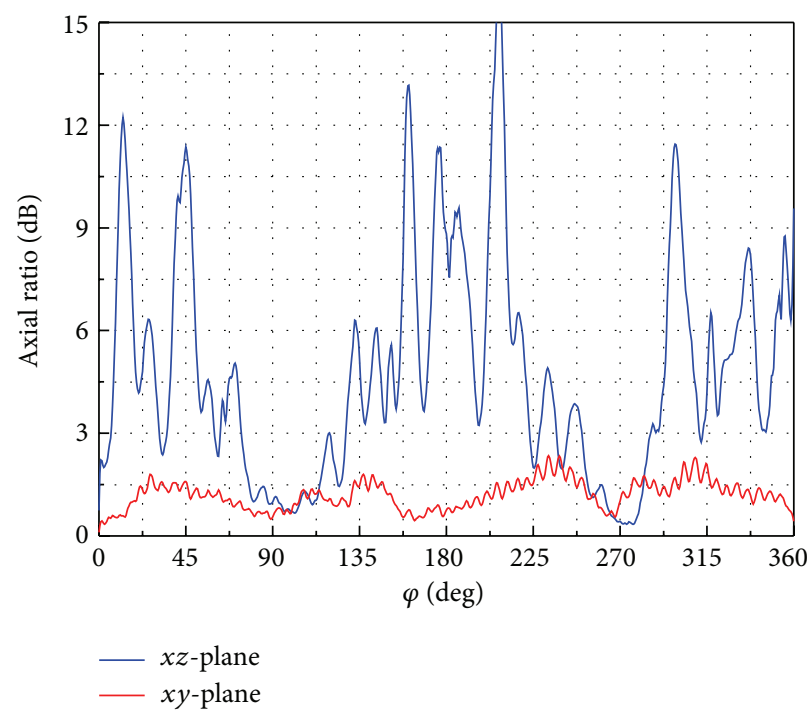

(b)

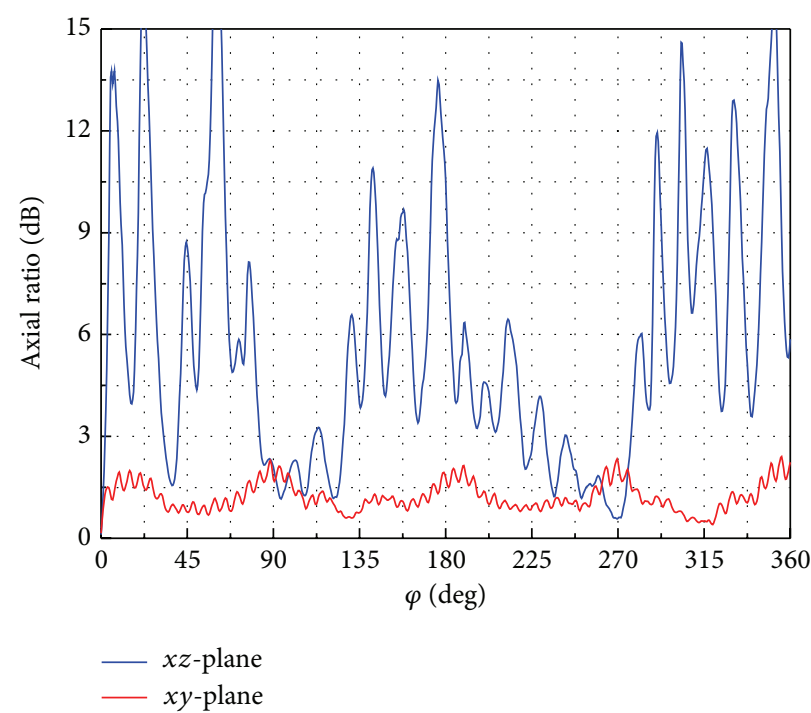

(c)

FIGURE 6: Measured axial ratio patterns in $x y$-plane and $x z$-plane. (a) $5.1 \mathrm{GHz}$, (b) $5.5 \mathrm{GHz}$, and (c) $5.9 \mathrm{GHz}$.

when excited RHCP port and LHCP port, respectively, are in the $x y$-plane (omnidirectional plane) and $x z$-plane at $5.1 \mathrm{GHz}, 5.5 \mathrm{GHz}$, and $5.9 \mathrm{GHz}$. From the measured radiation pattern results of $x y$-plane, the proposed antenna performs a good omnidirectional character. It is noted that the measured radiation patterns have the same trend with the simulated radiation patterns, but there are still a few discrepancies between measured results and simulated results. The reasons for this difference are mainly because of the machine error and the imprecision of the medium. It can be also seen from cross-polarization results that the cross-polarization is more than $15 \mathrm{~dB}$ lower than the copolarization in both $x y$-plane and $x z$-plane. The measured axial ratio patterns that only excited LHCP port in $x y$-plane and $x z$-plane at $5.1 \mathrm{GHz}$, $5.5 \mathrm{GHz}$, and $5.9 \mathrm{GHz}$ are shown in Figure 6. It can be seen that the axial ratio is below $3 \mathrm{~dB}$ in the omnidirectional plane within the operation bandwidth. Comparing the radiation patterns and axial ratio patterns results, the axial ratio is lower than $3 \mathrm{~dB}$ during the half power beam width. When only excited the RHCP port, the axial ratio is below $3 \mathrm{~dB}$ in the omnidirectional plane which is similar as Figure 6 shows. It can be noted that the axial ratio patterns in the $x y$-plane are small ripples. From the generation principle of the dual CP wave as analyzed in Section 2.2, the axial ratio in the vertical direction with the slot pairs will be the best and the value will be the minimum, while in the other nonvertical direction with the slot pairs the axial ratio will deteriorate. Because four slot pairs are arranged around the axis of coaxial cylinder in the out conductor, the slot pairs are noncoplanar. When rotated around the axis of the antenna, the axial ratio pattern in the $x y$-plane will be small rippled almost regularly as the symmetrical antenna structure in different $\varphi$ directions.

The measured gain and average axial ratio in the omnidirectional plane results are shown in Figure 7. It can be seen 


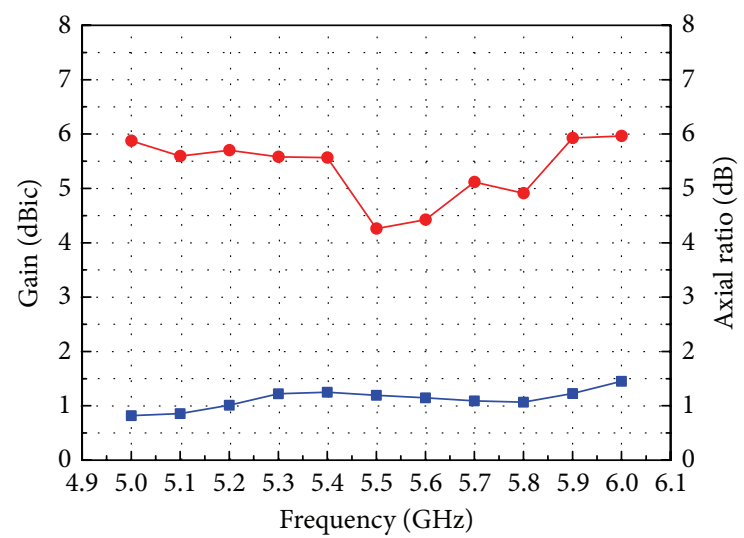

$\rightarrow$ Axial ratio

FIGURE 7: Measured gain and average axial ratio in the omnidirectional plane.

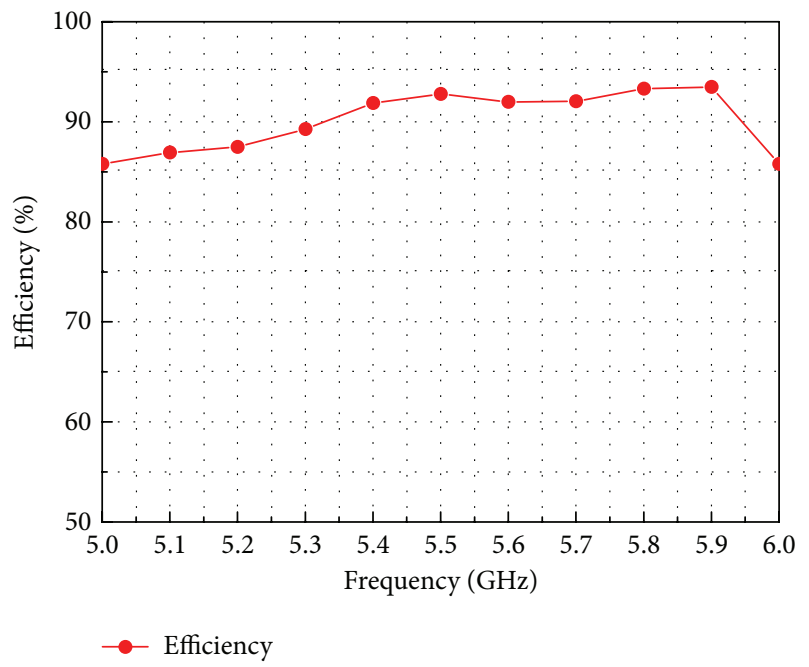

Figure 8: The total efficiency results of the proposed antenna.

that the gain is from $4 \mathrm{dBic}$ to $6 \mathrm{dBic}$ within $5.0 \sim 6.0 \mathrm{GHz}$, and the average axial ratio in $x y$-plane is below $1.5 \mathrm{~dB}$ within the bandwidth. Figure 8 shows the simulated total efficiency of the proposed antenna. It can be noted from the efficiency results that the total efficiency of the antenna from 5.1 to $5.9 \mathrm{GHz}$ is from $86.9 \%$ to $93.5 \%$, so the antenna works with very high efficiency within its operation bandwidth.

\section{Conclusion}

An omnidirectional dual CP antenna is presented in this paper. The antenna achieves the omnidirectional dual CP property by arranging perpendicular slot pairs around and along the coaxial cylinder axis in the out conductor. From the measured results, the antenna provides a bandwidth of $16.4 \%$ from 5.05 to $5.95 \mathrm{GHz}$ with an isolation higher than $15 \mathrm{~dB}$ and return loss higher than $10 \mathrm{~dB}$. Within the bandwidth, the gain is $4 \mathrm{dBic} \sim 6 \mathrm{dBic}$ and the average axial ratio is lower than
$1.5 \mathrm{~dB}$. The antenna works with high efficiency and performs good omnidirectional character. The cross-polarization is more than $15 \mathrm{~dB}$ lower than the copolarization in both $x y$ plane and $x z$-plane. The antenna is perfectly symmetric structure, so it is easy to change the working frequency band by changing the size of the antenna. For the omnidirectional coverage property and dual CP property, the antenna is valuable in the RF receivers.

\section{Conflict of Interests}

The authors declare that there is no conflict of interests regarding the publication of this paper.

\section{Acknowledgments}

This work was supported by the National Natural Science Foundation $(61201058,61471240)$, the Research and Innovation Project of Shanghai Education Commission (12Z112030001), and the Scientific Research Foundation for Returned Overseas Chinese Scholars, State Education Ministry, and the Project of SMC Excellent Young Faculty.

\section{References}

[1] W. Croswell and C. Cockrell, "An omnidirectional microwave antenna for use on spacecraft," IEEE Transactions on Antennas and Propagation, vol. 17, no. 4, pp. 459-466, 1969.

[2] K. Sakaguchi and N. Hasebe, "A circularly polarized omnidirectional antenna," in Proceedings of the 8th International Conference on Antennas and Propagation (ICAP '93), pp. 477480, 1993.

[3] C. Y. Yu, T. H. Xu, and C. J. Liu, "Design of a novel UWB omnidirectional antenna using particle swarm optimization," International Journal of Antennas and Propagation, vol. 2015, Article ID 303195, 7 pages, 2015.

[4] A. Narbudowicz, X. L. Bao, and M. J. Ammann, "Miniaturization of omnidirectional circularly polarized antennas," in Proceedings of the 8th European Conference on Antennas and Propagation (EuCAP '14), pp. 2919-2923, April 2014.

[5] M. Khalily, M. R. Kamarudin, M. Mokayef, and M. H. Jamaluddin, "Omnidirectional circularly polarized dielectric resonator antenna for 5.2-GHz WLAN applications," IEEE Antennas and Wireless Propagation Letters, vol. 13, pp. 443-446, 2014.

[6] X. D. Bai, X. L. Liang, M. Z. Li, B. Zhou, J. Geng, and R. Jin, "Dual-circularly polarized conical-beam microstrip antenna," IEEE Antennas and Wireless Propagation Letters, vol. 14, pp. 482-485, 2015

[7] D. Yu, S.-X. Gong, Y.-T. Wan, and W.-F. Chen, "Omnidirectional dual-band dual circularly polarized microstrip antenna Using $\mathrm{TM}_{01}$ and $\mathrm{TM}_{02}$ modes," IEEE Antennas and Wireless Propagation Letters, vol. 13, pp. 1104-1107, 2014.

[8] X.-L. Quan and R.-L. Li, "Broadband dual-polarized omnidirectional antennas," in Proceedings of the IEEE Antennas and Propagation Society International Symposium (APSURSI '12), pp. 1-2, IEEE, Chicago, Ill, USA, July 2012.

[9] B. Zhou, J. P. Geng, X. D. Bai, L. Duan, X. Liang, and R. Jin, "An omnidirectional circularly polarized slot array antenna with high gain in a wide bandwidth," IEEE Antennas and Wireless Propagation Letters, vol. 14, pp. 666-669, 2015. 
[10] K. Iigusa, T. Teshirogi, M. Fujita, S.-I. Yamamoto, and T. Ikegami, "A slot-array antenna on a coaxial cylinder with a circularly polarized conical beam," Electronics and Communications in Japan Part I: Communications, vol. 83, no. 3, pp. 74-87, 2000.

[11] M. E. Bialkowski and P. W. Davis, "Linearly polarized radialline slot-array antenna with a broadened beam," Microwave and Optical Technology Letters, vol. 27, no. 2, pp. 98-101, 2000. 

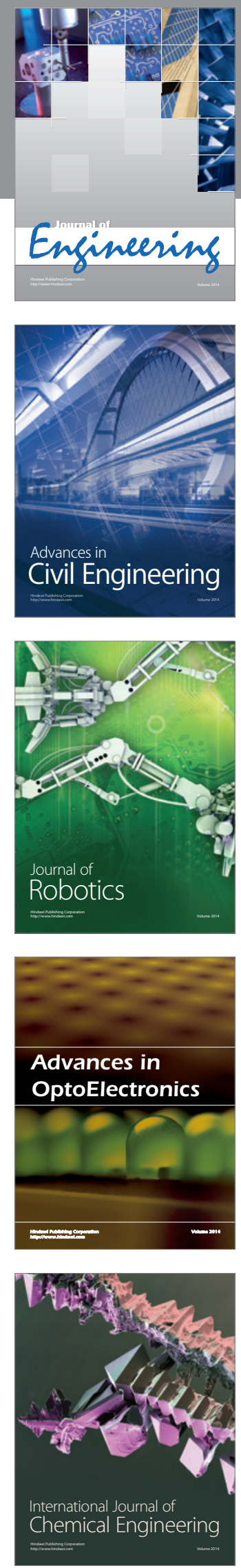

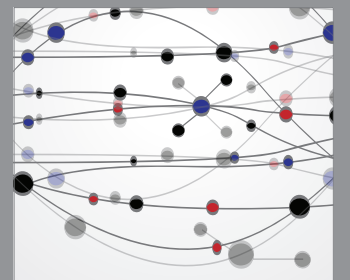

The Scientific World Journal
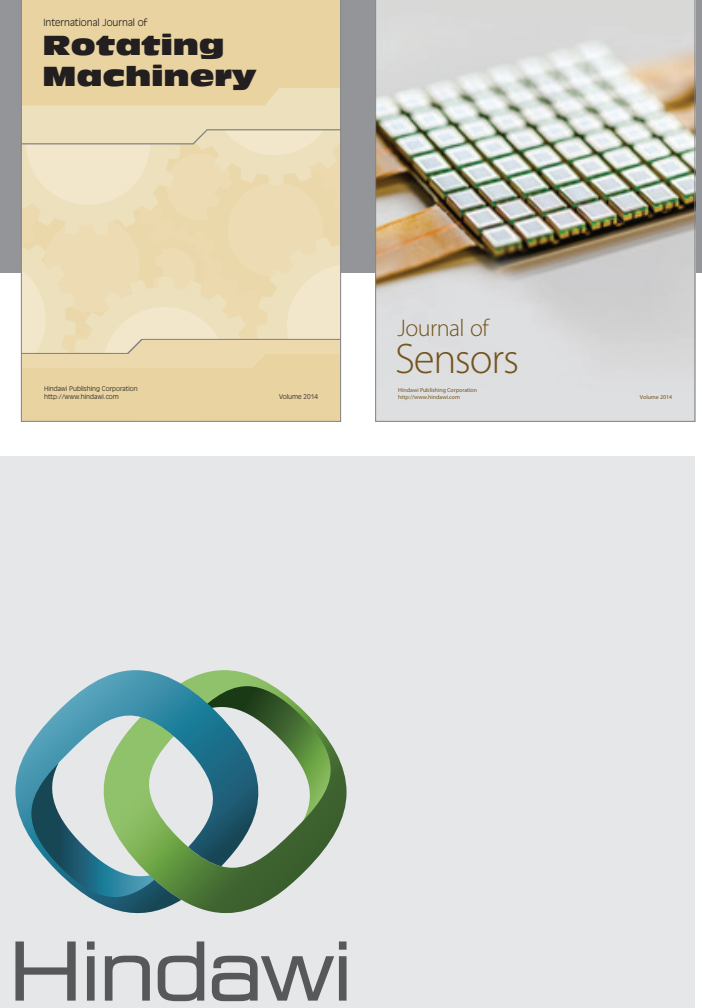

Submit your manuscripts at http://www.hindawi.com
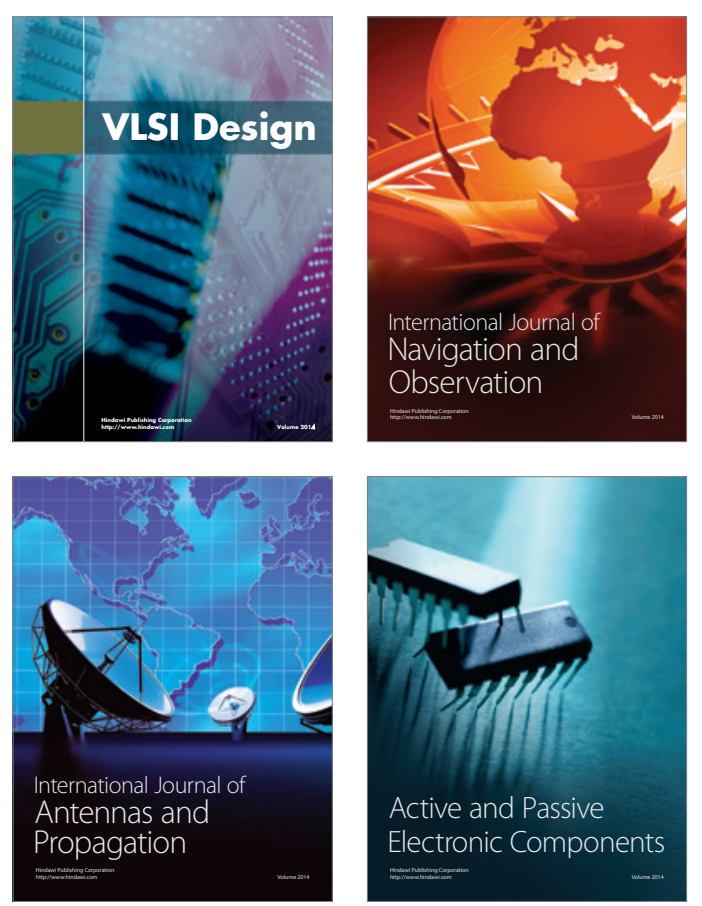
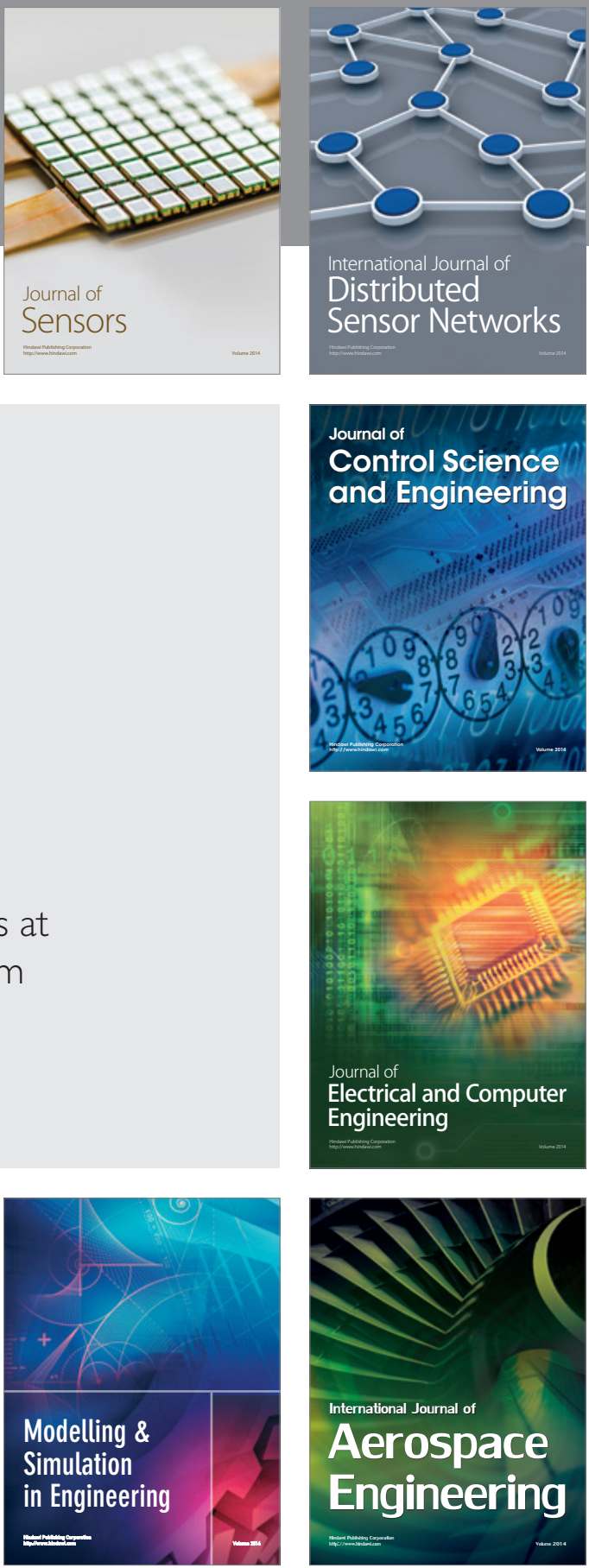

Journal of

Control Science

and Engineering
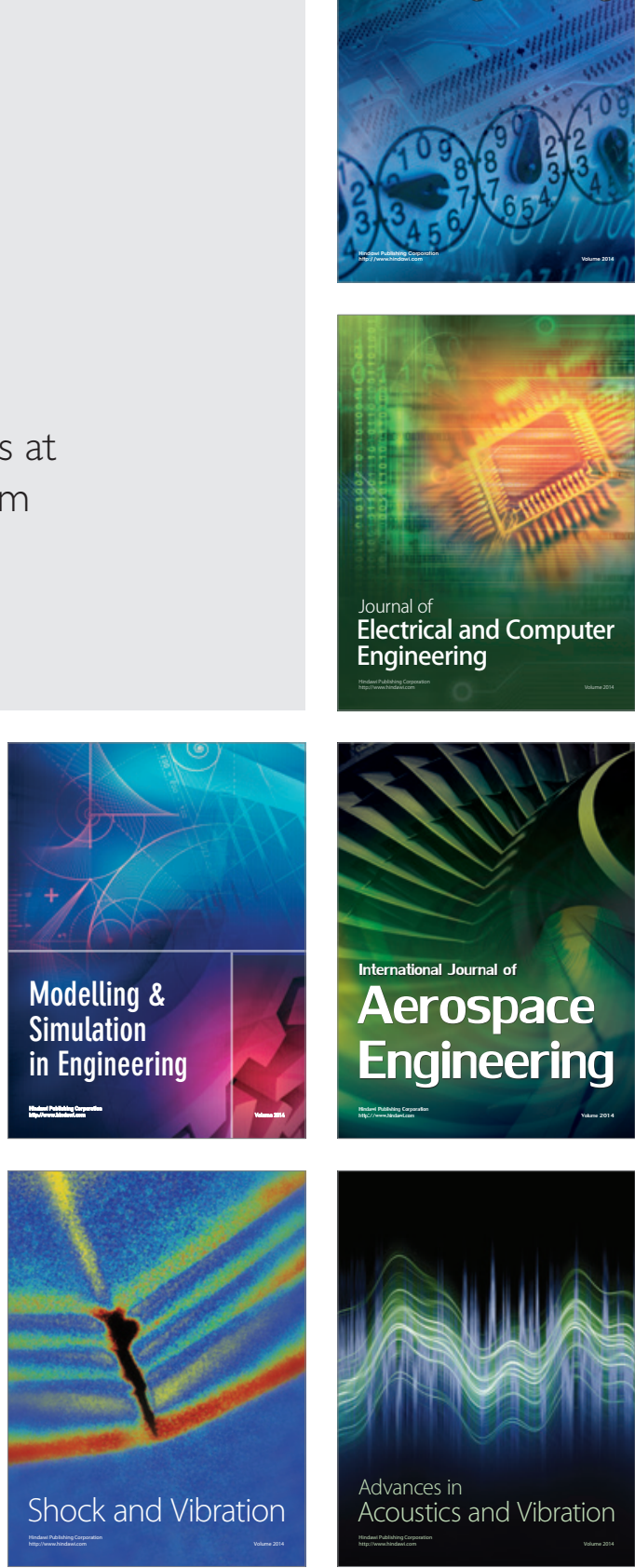